Case report

\title{
Deciduoma of the ovary complicating pregnancy
}

\author{
Rangaswamy Vedavalli, Satish Korah Kuruvila
}

Pondicherry Institute of Medical Sciences, Pondicherry, India

Received 16 August 2013, Accepted 1 October 2013

(C) 2013, Vedavalli R., Kuruvila S.K.

(C) 2013, Russian Open Medical Journal

\begin{abstract}
A 24 yr old Primigravida who conceived spontaneously came with two months amenorrhea for confirmation of pregnancy and routine checkup. Her 1st trimester scan, showed a single intra - uterine viable pregnancy of 8-9 weeks, with a right adnexal mass of about $10.7 \times 7.7 \times 10.3 \mathrm{~cm}$ with solid and cystic areas with internal echoes. In view of the size of the tumor and its varying echogenicity, pathological condition of the ovary was suspected. She reported at 24 weeks with abdominal pain. Partial torsion was suspected and Laprotomy was done. Right ovary was enlarged to $10 \mathrm{~cm}$ with varying consistency. Right ovariotomy was done. Postoperative period was uneventful. Histopathological examination showed extensive decidual changes in the ovary, forming tumor like lesions in the cystic areas. The pregnancy progressed without any complication. At term, she delivered a healthy female baby. The case is being presented for its extreme rarity.
\end{abstract}

Keywords: deciduoma ovary, tumour, pregnancy

Cite as Vedavalli R, Kuruvila SK. Deciduoma of the ovary complicating pregnancy. Russian Open Medical Journal $2014 ; 3: 0105$.

Correspondence to Dr. Kuruvila Satish. Address: Department of Gynecology, Pondicherry Institute of Medical Sciences, Pondicherry, India, 605014.

Phone: +919047016940. E-mail: skuruvila@gmail.com

\section{Case report}

24 years old Mrs. $X$ came with a history of two months amenorrhoea for confirmation of pregnancy. She did not have any complaints. She has been married for 4 years. Her periods were regular and normal. Her last menstrual period was on June $12^{\text {th }}$ and expected date of delivery was on March $19^{\text {th }}$. She conceived spontaneously. There was no relevant medical or surgical history.

She was sent for routine first trimester scan. Routine scan showed a viable Intra uterine pregnancy of 9 weeks duration and a Right adnexal mass of about $10.7 \times 7.1 \times 10.3 \mathrm{~cm}$ with predominantly cystic and solid components. In view of the size of the tumor and varying echogenicity, a pathological condition of the ovary was suspected. As pregnancy was early, and she did not have any complaints. She was advised to come one month later, for removal of the mass.

She reported two months later. Repeat scan showed normal growth of the foetus of about 16 wks with Rt. Adnexal mass with the same finding as before. She had no complaints. A provisional diagnosis of Dermoid Endometrioma was made and laprotomy was planned, for the next day.

She reported eight weeks later with pain on the right side of the abdomen. Scan showed normal growth of the fetus and the mass in the right adnexa did not show any changes. Her vitals were stable. Fetus was normal. Partial Torsion was suspected and hence proceeded with laprotomy.

Abdomen was opened by mid line sub umbilical lesion. Uterus was enlarged to 24-26 wks size. Right ovary was enlarged to $10 \mathrm{~cm}$ and was mildly adherent posteriorly. Accessibility to the pedicle was difficult Adhesions were released by careful dissection. Right
Ovary was removed as much as possible. Haemostasis was obtained. Abdomen was closed. Patient was on tocolytic agent for 48 hrs. No other specific treatment was given. Postoperative period was uneventful.

The left ovary was not visualized due to technical difficulty. Cut section of the ovarian mass showed multi locular cyst filled with light brown colored liquid and solid areas. Histopathological examination showed extensive decidual changes in the ovary, forming tumor like lesion with cystic areas.

Pregnancy progressed without any complaints. She delivered an alive female baby of $2.83 \mathrm{kgs}$ by elective lower segment caesarian section at term for placenta praevia type II and cord around.

\section{Discussion}

Deciduoma of the ovary simulating neoplasm is rare. The ovarian stromal cells has the potential to differentiate presumably by a process of metaplasia into a variety of other mesenchymal cell types, most commonly decidual [1], in response to elevated progesterone level. An ovarian decidual reaction usually represents the response of indigenous stromal cells to hormonal milieu of pregnancy. They may occur singly or as nodules, rarely as large a nodule as in our case.

Three cases have been reported in the literature so far. In the first case ovary showed extensive decidual reaction [2]. In the second case there was an ovarian mass [3]. In the third case there was extensive decidual reaction in the peritoneal cavity with hemoperitoneum necessitating emergency laprotomy [4]. 
Conflict of interest: none declared.

\section{References}

1. Bersch W, Alexy E, Heuser HP, Staemmler HJ. Ectopic deciduas formation in the ovary (so-called deciduoma). Virchows Arch Abt Path Anat 1973; 360: 173-177.

2. Satyanarayana S, Bohre JK. Ovarian granulosa cell "tumorlet" and mature follicles with ectopic decidua in pregnancy - a case report. Indian J Pathol Microbiol 2001; 44(2): 149-150. (PMID: 11883133)

3. Clement PB. Tumor-like lesions of the ovary associated with pregnancy. Int J Gynecol Pathol 1993; 12(2): 108-115. (PMID: 8463033)

4. Ekabua JK, liam IH, Anglio EEJ, Eterk SJ, Eiuana AU, Jubrin JP. Extrauterine decidual reaction in pregnancy presenting as an acute abdomen: a case report. Tropical Journal of Obstetrics and Gynaecology 2001, 18(2): 91-92. (doi: 10.4314/tjog.v18i2.14438)

Authors:

Rangaswamy Vedavalli - B.Sc., MD., D.G.O., M.N.A.M.S., Formerly Professor of Obstetrics and Gynecology, Pondicherry Institute of Medical Sciences, Pondicherry, India;

Satish Korah Kuruvila - M.B.B.S., D.G.O., Dip.N.B., Professor of Obstetrics and Gynecology, Pondicherry Institute of Medical Sciences, Pondicherry, India. 